\title{
Thermodynamics and Reaction Kinetics of Symmetric Vitrimers based on Dioxaborolane Metathesis
}

\author{
Shilong Wu, Huanhuan Yang, Wen-Sheng Xu, Quan Chen*
}

State Key Laboratory of Polymer Physics and Chemistry, Changchun Institute of Applied Chemistry, Chinese Academy of Sciences, Renmin St 5625, Changchun 130022, China

*: All correspondence should be sent to qchen@ ciac.ac.cn

Table of contents:

1. Synthesis and Characterization

1.1 Synthesis and characterization of monomer 2,3-dioxaborolanepropyl methacrylate

1.2 Synthesis and characterization of monomer 4-vinylphenyl-2,3-dioxaborolanepropyl

1.3 RAFT copolymerization of precursors A- $F_{\mathrm{A}}$

1.4 RAFT copolymerization of precursors B- $F_{\mathrm{B}}$

1.5 Boron elemental analysis

2. X-ray Scattering Profiles

3. DSC Traces

4. Linear Viscoelasticity (LVE)

4.1 Stress relaxation measurements of vitrimer samples

4.2 Frequency sweep measurements of A-2.6/B- $F_{\mathrm{B}}$ samples

4.3 Shift factors of A-2.6/B- $F_{\mathrm{B}}$ samples 


\section{Synthesis and Characterization}

1.1. Synthesis and characterization of monomer 2,3-dioxaborolanepropyl methacrylate

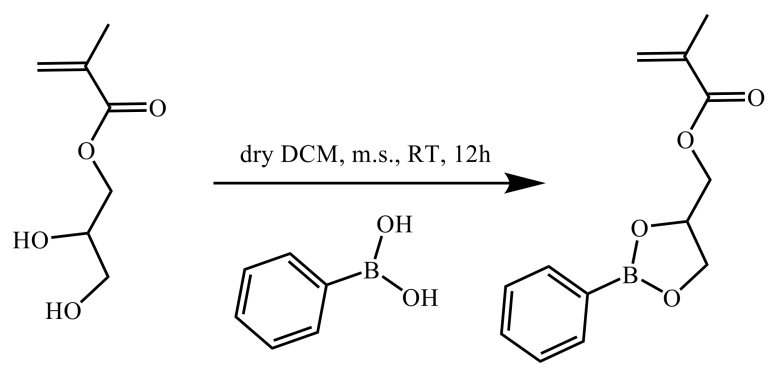

Scheme S1. The synthesis route of 2,3-dioxaborolanepropyl methacrylate

Phenylboronic acid $(0.02 \mathrm{~mol})$ and 2,3-dihydroxypropyl methacrylate $(0.02 \mathrm{~mol})$ were stirred in $100 \mathrm{~mL}$ dry DCM with $15 \mathrm{~g}$ molecular sieves in a flask for $12 \mathrm{~h}$ at room temperature. The process of the reaction was monitored by the ${ }^{1} \mathrm{H}$ NMR and ${ }^{13} \mathrm{C}$ NMR spectroscopy. After completion of the reaction, a raw product was purified through filtering, centrifuging, and separating on silica gel using column chromatography $[\mathrm{V}($ ethyl acetate $): \mathrm{V}(\mathrm{n}$-hexane $)=1: 30]$ to obtain the final product, a colorless oil sample. The product was characterized by ${ }^{1} \mathrm{H}$ NMR and ${ }^{13} \mathrm{C}$ NMR spectroscopy. The results are shown in Figures S1 and S2, respectively.

${ }^{1} \mathrm{H}$ NMR (400 MHz, $\mathrm{CDCl}_{3}$ ): $\delta(\mathrm{ppm})$ 7.29-7.75 (m, 5H), $6.03(\mathrm{~s}, 1 \mathrm{H}), 5.48(\mathrm{~s}, 1 \mathrm{H}), 4.74-4.80$ $(\mathrm{m}, 1 \mathrm{H}), 4.37-4.41(\mathrm{t}, 1 \mathrm{H}), 4.20-4.31(\mathrm{~m}, 1 \mathrm{H}), 4.11-4.14(\mathrm{dd}, 1 \mathrm{H}), 1.84(\mathrm{~s}, 3 \mathrm{H}) .{ }^{13} \mathrm{C}$ NMR $(100$ $\left.\mathrm{MHz}, \mathrm{CDCl}_{3}\right): \delta(\mathrm{ppm}) 167.1,135.8,134.9,134.9,131.4,127.6,127.6,126.4,74.9,67.8,65.6$, 18.2. The boron-bound carbon was not detected due to quadrupolar relaxation. 


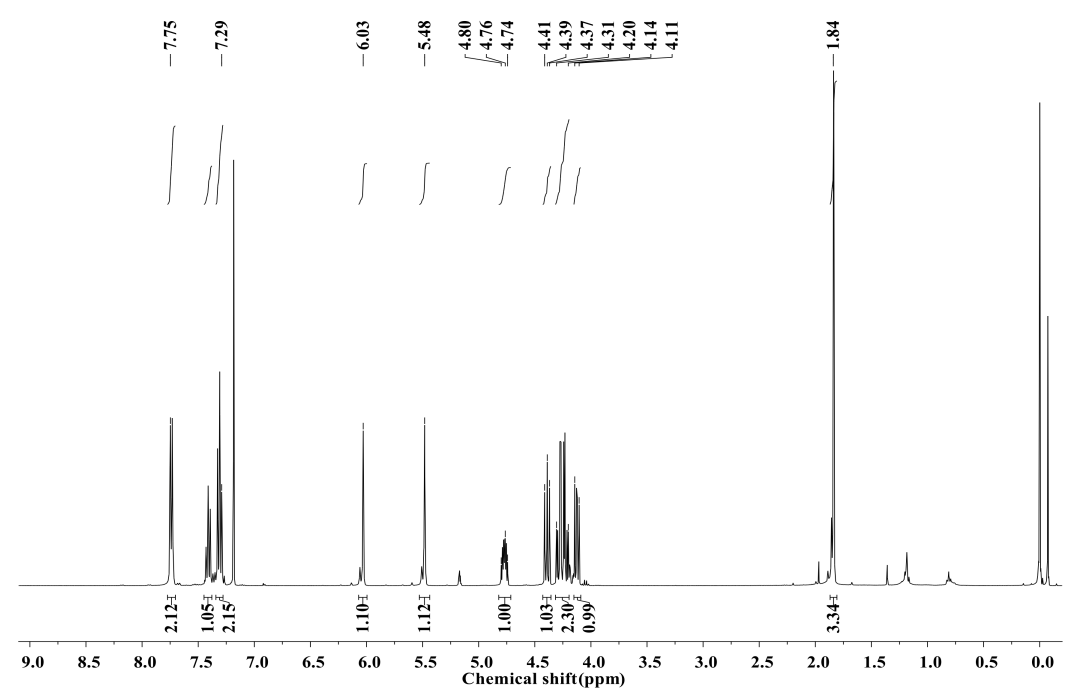

Figure S1. ${ }^{1} \mathrm{H}$ NMR spectrum of 2,3-dioxaborolanepropyl methacrylate.

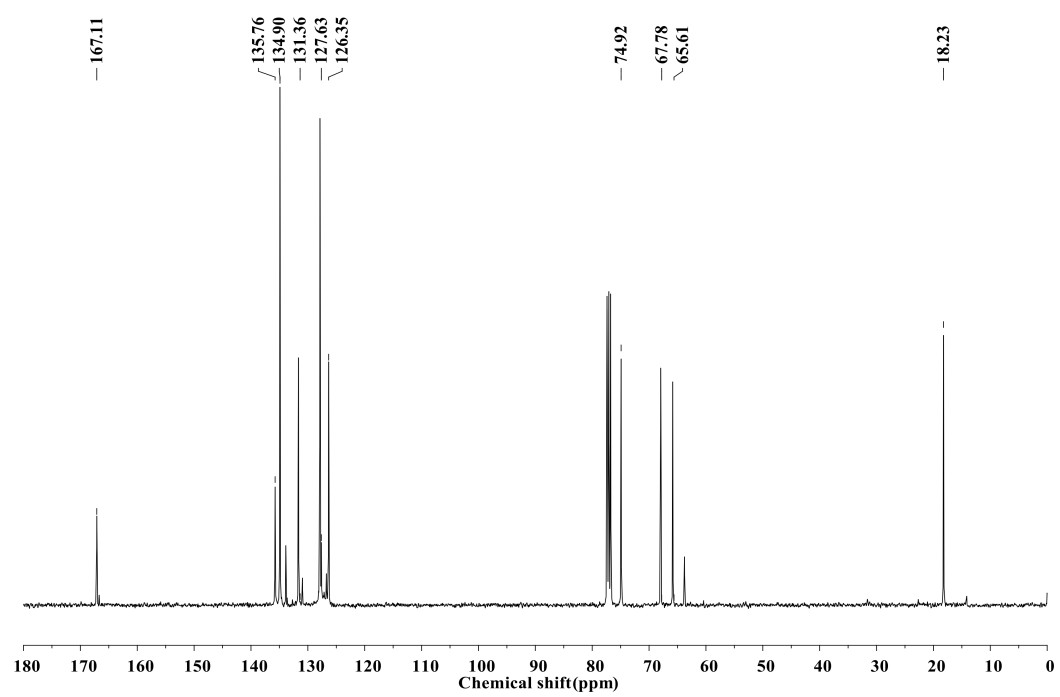

Figure S2. ${ }^{13} \mathrm{C}$ NMR spectrum of 2,3-dioxaborolanepropyl methacrylate.

1.2. Synthesis and characterization of monomer 4-vinylphenyl-2,3-dioxaborolanepropyl<smiles>C=Cc1ccc(B(O)O)cc1</smiles>

dry DCM, m.s., RT, $12 \mathrm{~h}$<smiles>C=Cc1ccc(B2OCC(C)O2)cc1</smiles>

Scheme S2. The synthesis route of 4-vinylphenyl-2,3-dioxaborolanepropyl 
1,2-propanediol $(0.02 \mathrm{~mol})$ and 4-vinylphenylboronic acid $(0.02 \mathrm{~mol})$ were stirred in $100 \mathrm{~mL}$ dry DCM with $15 \mathrm{~g}$ molecular sieves in a flask for $12 \mathrm{~h}$ at room temperature. The product was purified and characterized in the same way as 2,3-dioxaborolanepropyl methacrylate. The ${ }^{1} \mathrm{H}$ NMR and ${ }^{13} \mathrm{C}$ NMR results of the product are shown in Figures S3 and S4, respectively.

${ }^{1} \mathrm{H}$ NMR (400 MHz, $\left.\mathrm{CDCl}_{3}\right): \delta(\mathrm{ppm})$ 7.34-7.71(dd, 4H), 6.62-6.69 (m, 1H), 5.72-5.77 (d, 1H), 5.21-5.24(d, $1 \mathrm{H}), 4.62-4.68(\mathrm{~m}, 1 \mathrm{H}), 4.35-4.39(\mathrm{~m}, 1 \mathrm{H}), 3.79-3.83(\mathrm{~m}, 1 \mathrm{H}), 1.33-1.36(\mathrm{~d}, 3 \mathrm{H}) .{ }^{13} \mathrm{C}$ NMR (100 MHz, $\left.\mathrm{CDCl}_{3}\right): \delta(\mathrm{ppm}) 140.4,136.9,135.1,135.1,125.7,125.7,115,73.8,72.6$, 21.8. The boron-bound carbon was not detected due to quadrupolar relaxation.
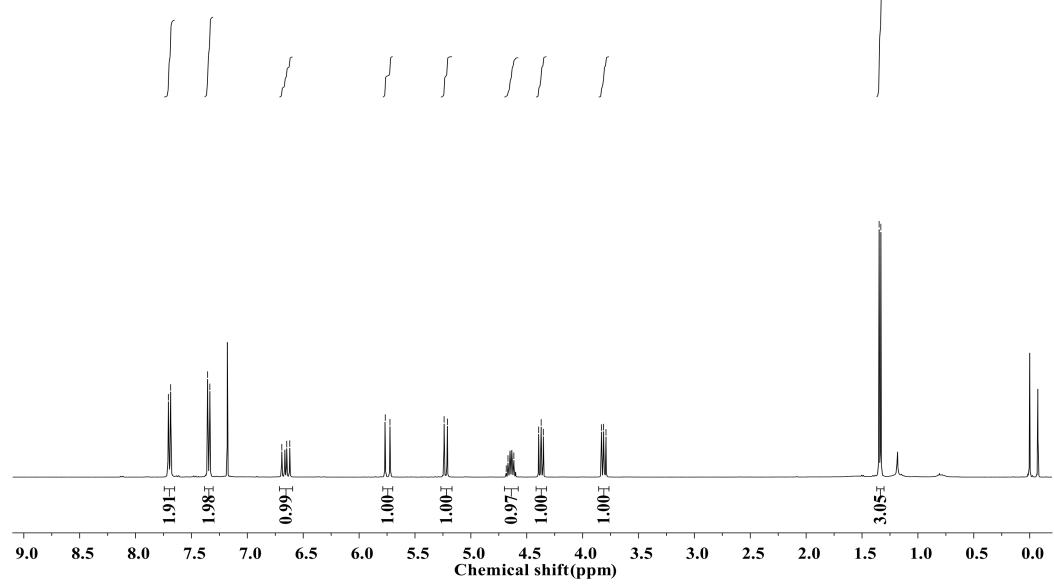

Figure S3. ${ }^{1} \mathrm{H}$ NMR spectrum of 4-vinylphenyl-2,3-dioxaborolanepropyl.
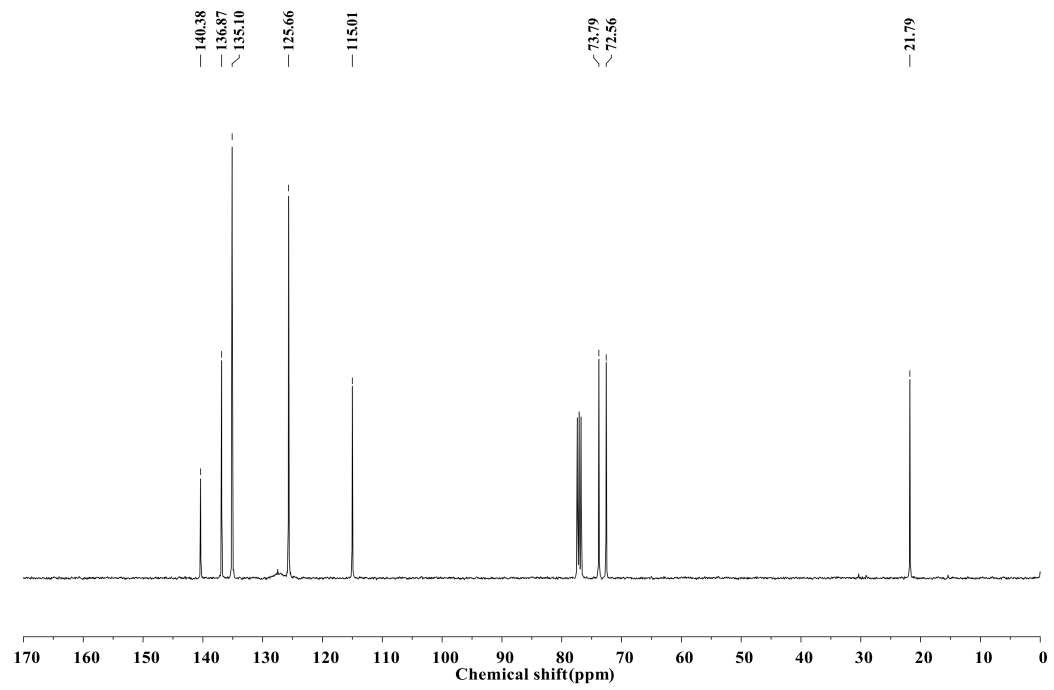

Figure S4. ${ }^{13} \mathrm{C}$ NMR spectrum of 4-vinylphenyl-2,3-dioxaborolanepropyl. 
1.3. RAFT copolymerization of precursors A-F $F_{\mathrm{A}}$ : poly (hexyl methacrylate-random-2,3dioxaborolanepropyl methacrylate)

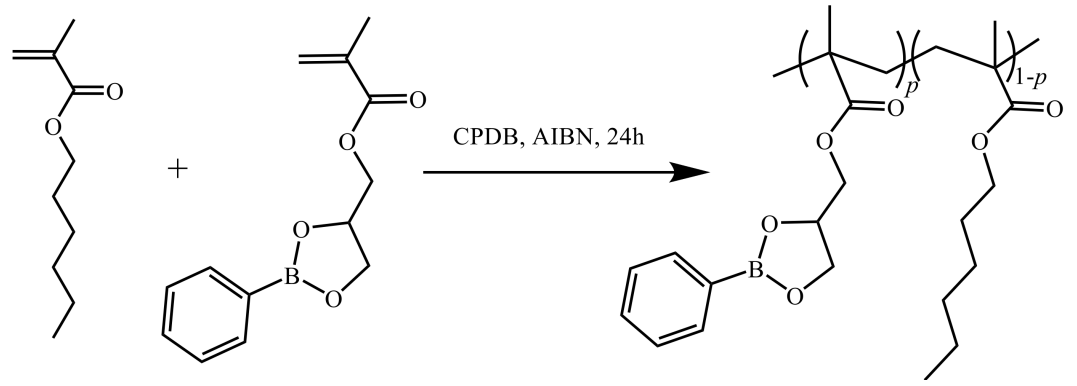

Scheme S3. The synthesis route of poly (hexyl methacrylate-random-2,3-dioxaborolanepropyl methacrylate)

To synthesize the precursors, poly (hexyl methacrylate-random-2,3-dioxaborolanepropyl methacrylate) $\left(\mathrm{A}-F_{\mathrm{A}}\right)$, predetermined molar ratios of purified monomers HMA and 2,3dioxaborolanepropyl methacrylate, the chain transfer agent CPDB and the polymerization initiator AIBN at fixed molar ratio of $[\mathrm{M}]:[\mathrm{CTA}]:[\mathrm{I}]=50: 1: 0.2$ were dissolved in anhydrous toluene $(5 \mathrm{~mL})$ in an ampoule. Before the reaction, three freeze-pump-thaw cycles were applied to remove oxygen from the solvent. During the reaction, the ampoule was bubbled with nitrogen and immersed into thermostatic oil bath thermo-stabilized at $70^{\circ} \mathrm{C}$. This condition appears to enable random copolymerization of monomers HMA and 2,3-dioxaborolanepropyl methacrylate. ${ }^{1}$ After $24 \mathrm{~h}$ polymerization, the reaction was completed and the ampoule was inserted into liquid nitrogen to quench the reaction. The product was poured into a large excess of methanol $(\sim 500 \mathrm{~mL})$ to precipitate $\mathrm{A}-F_{\mathrm{A}}$, where $F_{\mathrm{A}}$ represents average number of monomer 2,3-dioxaborolanepropyl methacrylate per precursor chain. After centrifuging, the precipitant was dissolved in $\mathrm{CHCl}_{3}$ and reprecipitated in excess of methanol $(\sim 500 \mathrm{~mL})$ for purification. 
The final products were dried in vacuum at $80^{\circ} \mathrm{C}$ for more than one week before preparing the vitrimer samples. In this process, six different precursor samples $\mathrm{A}-F_{\mathrm{A}}$ were synthesized, with $F_{\mathrm{A}}=1.2,2.1,2.6,3.5,5.7,11$. The results of the ${ }^{1} \mathrm{H}$ NMR and GPC for precursor samples A$F_{\mathrm{A}}$ were shown in Figures $\mathrm{S} 5$ and $\mathrm{S} 6$, respectively.

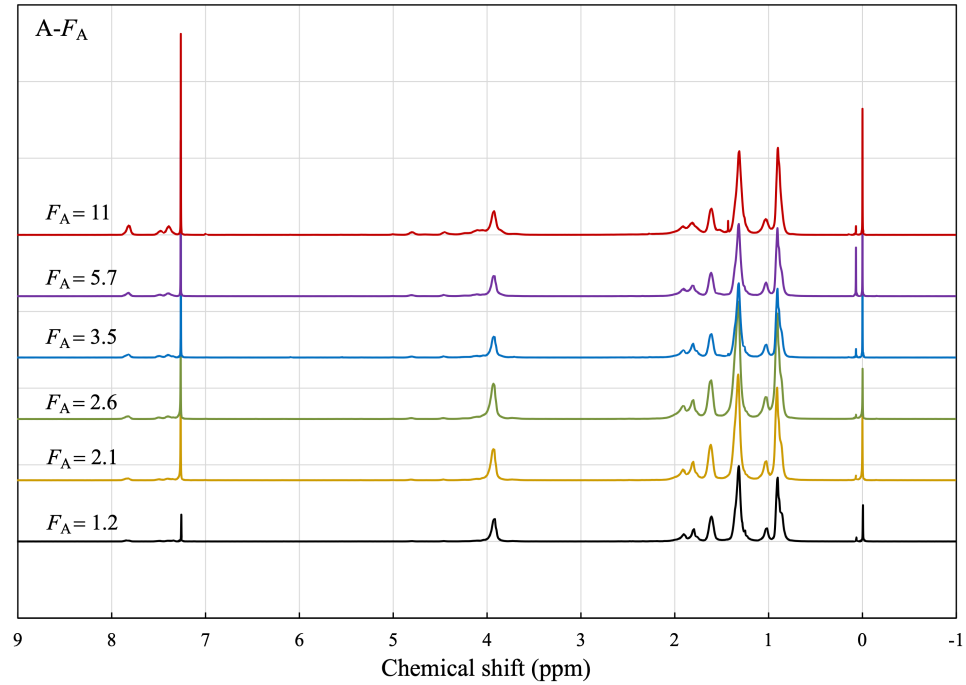

Figure S5. ${ }^{1} \mathrm{H}$ NMR spectra of precursor samples A- $F_{\mathrm{A}}$ with $F_{\mathrm{A}}=1.2,2.1,2.6,3.5,5.7,11$.

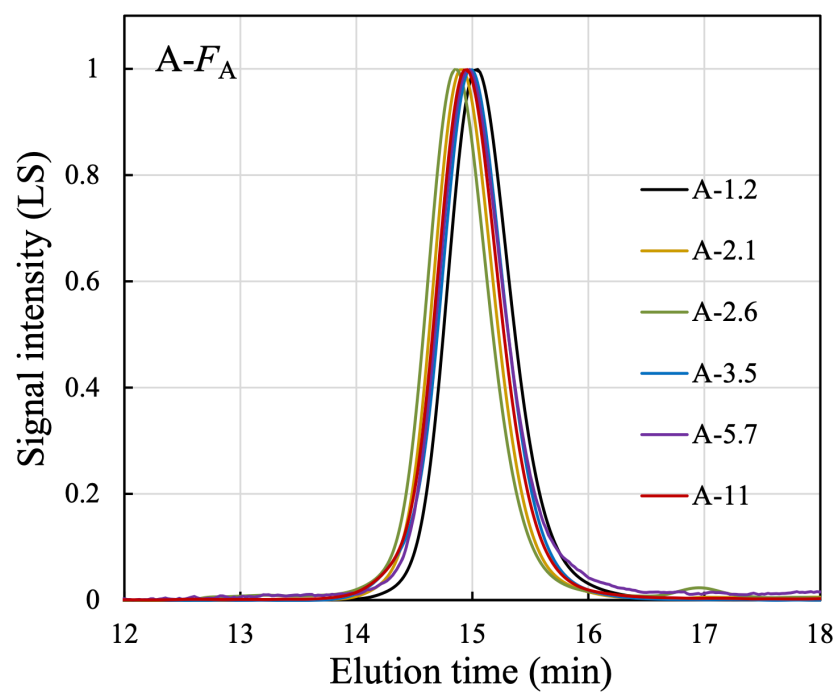

Figure S6. GPC spectra of precursor samples A- $F_{\mathrm{A}}$ with $F_{\mathrm{A}}=1.2,2.1,2.6,3.5,5.7,11$. 
1.4. RAFT copolymerization of precursors B- $F_{\mathrm{B}}$ : poly (hexyl methacrylate-random-4vinylphenyl-2,3-dioxaborolanepropyl)

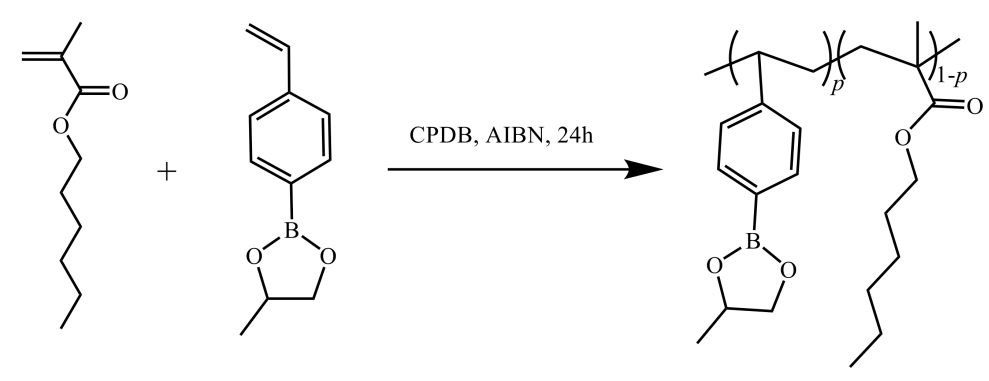

Scheme S4. The synthesis route of poly (hexyl methacrylate-random-4-vinylphenyl-2,3dioxaborolanepropyl)

To synthesize the precursors, poly (hexyl methacrylate-random-4-vinylphenyl-2,3dioxaborolanepropyl) (B- $\left.F_{\mathrm{B}}\right)$, predetermined molar ratios of purified monomers HMA, 4vinylphenyl-2,3-dioxaborolanepropyl), the chain transfer agent CPDB and the polymerization initiator AIBN at fixed molar ratio of $[\mathrm{M}]$ : $[\mathrm{CTA}]:[\mathrm{I}]=$ 50:1:0.2 were dissolved in anhydrous toluene $(5 \mathrm{~mL})$ in an ampoule. Before the reaction, three freeze-pump-thaw cycles were applied to remove oxygen from the solvent. The polymerization, purification and drying procedures of $\mathrm{B}-F_{\mathrm{B}}$ were the same as those of $\mathrm{A}-F_{\mathrm{A}}$. In this study, four precursor samples $\mathrm{B}-F_{\mathrm{B}}$ were synthesized, with $F_{\mathrm{B}}=1.6,2.3,3.4,7.0$. The results of the ${ }^{1} \mathrm{H}$ NMR and GPC precursor samples B- $F_{\mathrm{B}}$ were shown in Figures $\mathrm{S} 7$ and $\mathrm{S} 8$, respectively. 


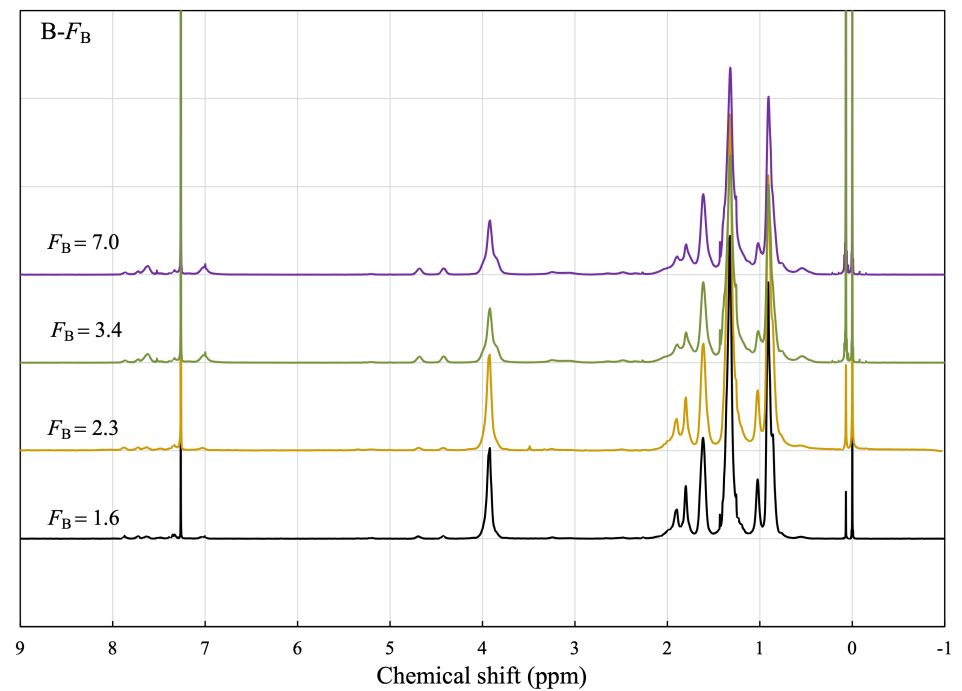

Figure S7. ${ }^{1} \mathrm{H}$ NMR spectra of precursor samples B- $F_{\mathrm{B}}$ with $F_{\mathrm{B}}=1.6,2.3,3.4,7.0$.

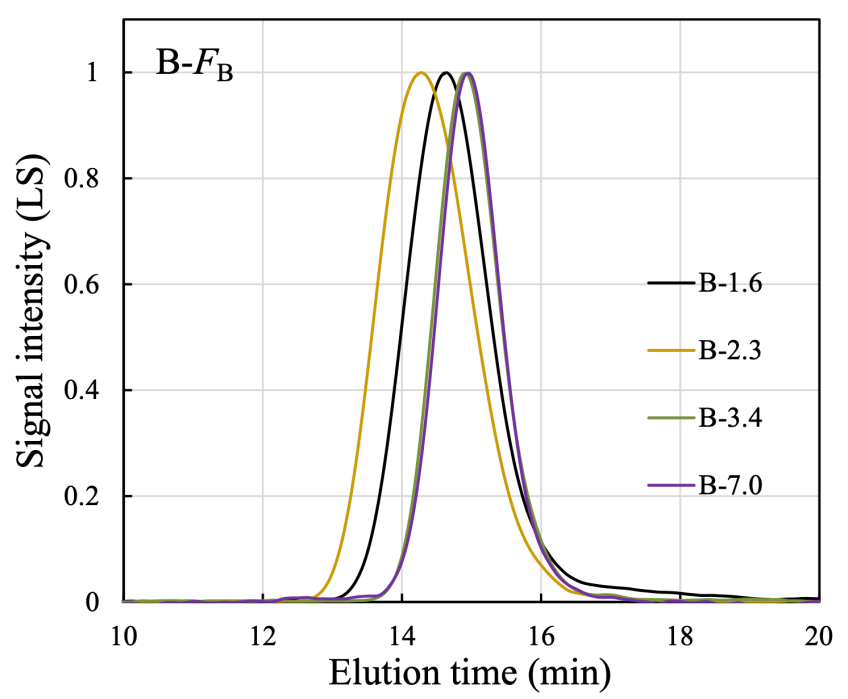

Figure S8. GPC spectra of precursor samples B- $F_{\mathrm{B}}$ with $F_{\mathrm{B}}=1.6,2.3,3.4,7.0$.

\subsection{Boron elemental analysis}

The two types of precursor samples, $\mathrm{A}-F_{\mathrm{A}}$ and $\mathrm{B}-F_{\mathrm{B}}$, were mixed with the molar ratio of 1:1 to prepare for the vitrimer samples. That is:

$\mathrm{A}-F_{\mathrm{A}}: \mathrm{B}-F_{\mathrm{B}}=m_{\mathrm{A}} / M_{\mathrm{n}, \mathrm{A}}: m_{\mathrm{B}} / M_{\mathrm{n}, \mathrm{B}}=1: 1$

where $m_{\mathrm{X}}$ and $M_{\mathrm{n}, \mathrm{X}}$ (with $\mathrm{X}=\mathrm{A}$ or $\mathrm{B}$ ) represent the feeding mass and number average molecular weight of the X component, respectively.

If all small molecules were removed in the vitrimer system, the expected Boron content [B] of the vitrimer sample becomes: 
$[\mathrm{B}]=m_{\mathrm{A}}\left[\mathrm{B}_{\mathrm{A}-\mathrm{FA}}\right] /\left(m_{\mathrm{A}}+m_{\mathrm{B}}\right) \quad$ if $m_{\mathrm{A}}\left[\mathrm{B}_{\mathrm{A}-\mathrm{FA}}\right]>m_{\mathrm{B}}\left[\mathrm{B}_{\mathrm{B}-\mathrm{FB}}\right]$

$[\mathrm{B}]=m_{\mathrm{B}}\left[\mathrm{B}_{\mathrm{B}-\mathrm{FB}}\right] /\left(m_{\mathrm{A}}+m_{\mathrm{B}}\right) \quad$ if $m_{\mathrm{A}}\left[\mathrm{B}_{\mathrm{A}-\mathrm{FA}}\right]<m_{\mathrm{B}}\left[\mathrm{B}_{\mathrm{B}-\mathrm{FB}}\right]$

where $m_{\mathrm{X}}$ and $\left[\mathrm{B}_{\mathrm{X}-\mathrm{FX}}\right]$ (with $\mathrm{X}=\mathrm{A}$ or $\mathrm{B}$ ) represent the feeding mass and the Boron content of the precursors $\mathrm{A}-F_{\mathrm{A}}$ or $\mathrm{B}-F_{\mathrm{B}}$ determined from ICP-OES, respectively. Please note the assumptions in these equations: (a) The reaction between $\mathrm{A}-F_{\mathrm{A}}$ and $\mathrm{B}-F_{\mathrm{B}}$ has been completed, and (b) all small-molecular byproduct B has been removed.

\section{X-ray Scattering Profiles}

WAXS profiles were collected using the Rigaku SmartLab X-ray diffractometer. The diffractometer was equipped with a copper target $(2.2 \mathrm{~kW})$, a CBO mirror $\left(\mathrm{K} \alpha_{1}\right.$ line, $\lambda=0.154$ $\mathrm{nm}$ ), a motorized sample stage, and a multi-slit scintillation detector. X-ray scattering patterns were recorded in a scattering angle $(2 \theta)$ ranging from $0.5^{\circ}$ to $25^{\circ}$ at a step-scan rate of $0.05^{\circ}$ per 5s. $\theta$ is half of the scattering angle, corresponding to the scattering vector $q=4 \pi \sin \theta / \lambda$ of 0.35-17.67 $\mathrm{nm}^{-1}$. SAXS experiments were performed using NanoSTAR-U (BRUKER AXS INC.) with $\mathrm{Cu} \mathrm{K} \alpha$ radiation (wavelength $\lambda=0.154 \mathrm{~nm}$ ). The generator was operated at $40 \mathrm{kV}$ and $650 \mu \mathrm{A}$. The sample-to-detector distance was $\mathrm{LSD}=1122 \mathrm{~mm}$, and two-dimensional SAXS patterns were obtained using a HI-STAR detector. All data were collected in a range of effective scattering vector $q$ from 0.08 to $1.9 \mathrm{~nm}^{-1}$. All measurements were conducted at room temperature, and the results are summarized in Figures S9 and S10.

Figure $\mathrm{S} 9$ compares the WAXS profiles of the vitrimer samples $\mathrm{A}-F_{\mathrm{A}} / \mathrm{B}-2.3$, where $F_{\mathrm{A}}=1.2$, 2.6, 5.7, 11 in panel (a), and A-2.6/B- $F_{\mathrm{B}}$, where $F_{\mathrm{B}}=1.6,2.3,3.4,7.0$ in panel (b). Two correlation peaks are seen for all vitrimer samples, i.e., the high- $q$ sidechain correlation peak at 
$q^{*}=13.03 \mathrm{~nm}^{-1}$ and the low- $q$ backbone-backbone correlation peak at $q^{*}=4.27 \mathrm{~nm}^{-1}$. The sidechain correlation distance $d=0.48 \mathrm{~nm}$ and the backbone-backbone correlation distance $d=$ $1.47 \mathrm{~nm}$ can be calculated according to Bragg's Law, $d=2 \pi / q^{*}$. These values are similar with two correlation peaks from pure PHMA in our previous work. ${ }^{2}$ No obvious difference is observed for these two peaks after the incorporation of the monomers containing the functional groups, probably owing to the small content of these monomers. No aggregates peak is observed at $q$ lower than the backbone-correlation peak, indicating that there is not microscopic phase separation in the system. This feature is further confirmed by the SAXS profiles shown in Figure S10.

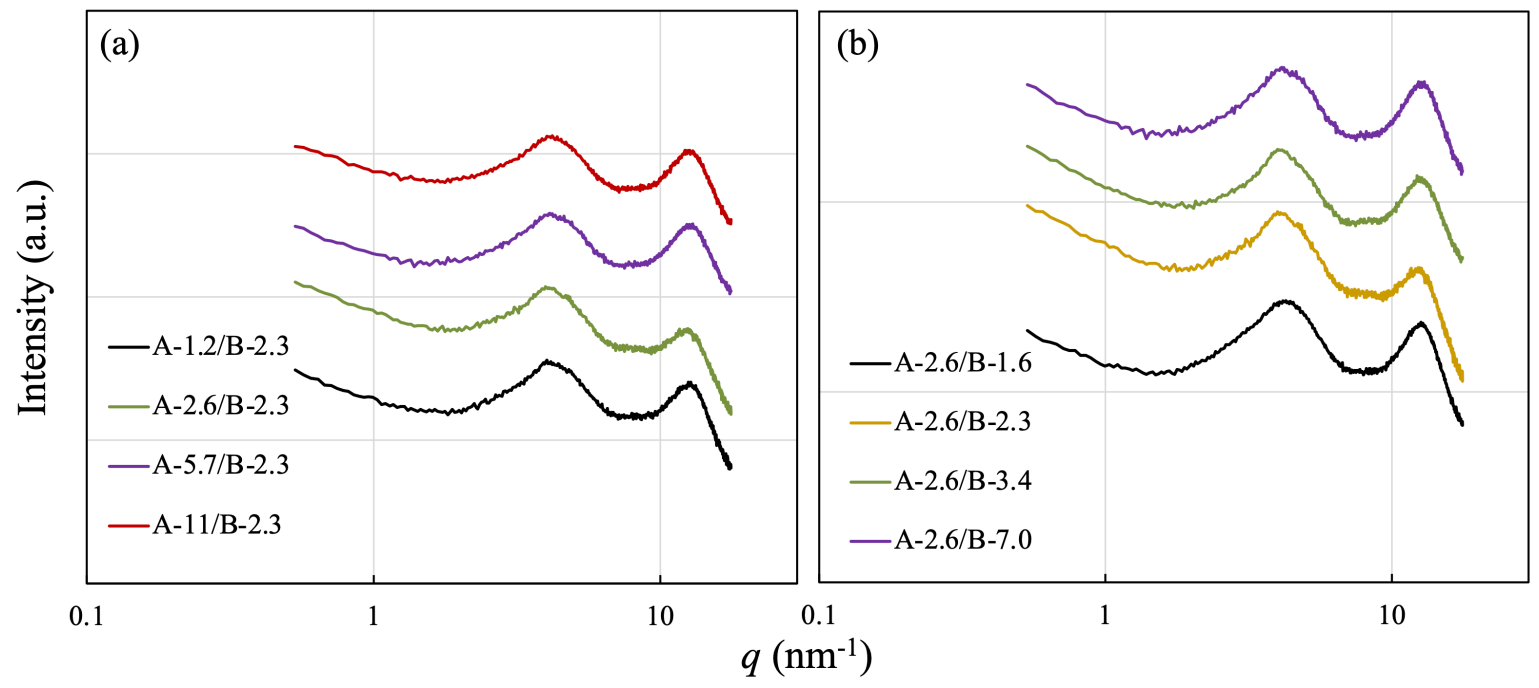

Figure S9. Comparison of WAXS profiles of vitrimer samples (a) A- $F_{\mathrm{A}} / \mathrm{B}-2.3$ with $F_{\mathrm{A}}=1.2,2.6,5.7,11$ and (b) A-2.6/B- $F_{\mathrm{B}}$ with $F_{\mathrm{B}}=1.6,2.3,3.4,7.0$. 


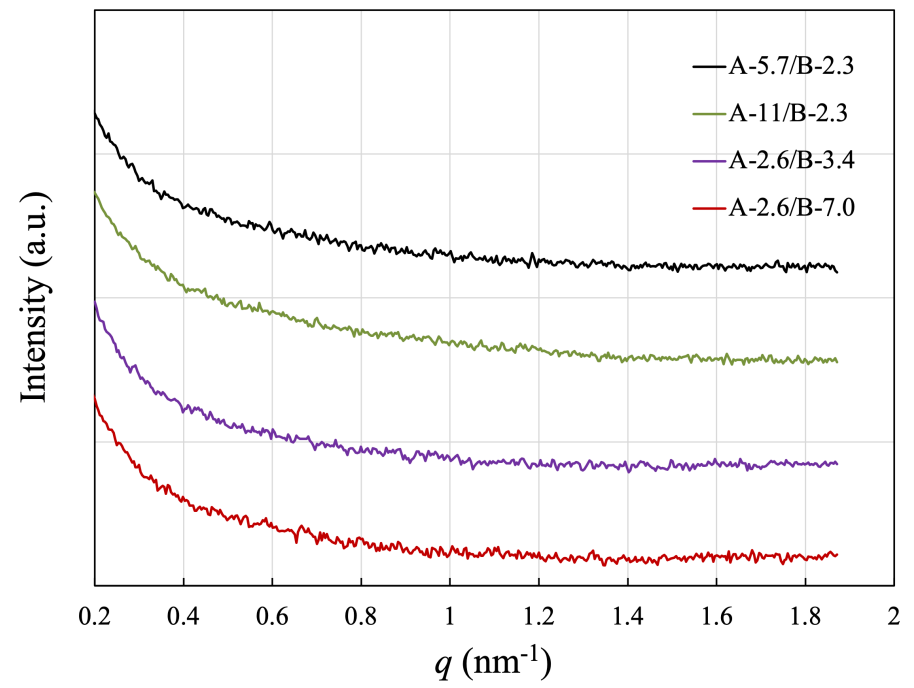

Figure S10. Comparison of SAXS profiles of vitrimer samples A- $F_{\mathrm{A}} / \mathrm{B}-2.3$ with $F_{\mathrm{A}}=5.7,11$ and A-2.6/B$F_{\mathrm{B}}$ with $F_{\mathrm{B}}=3.4,7.0$.

\section{DSC Traces}

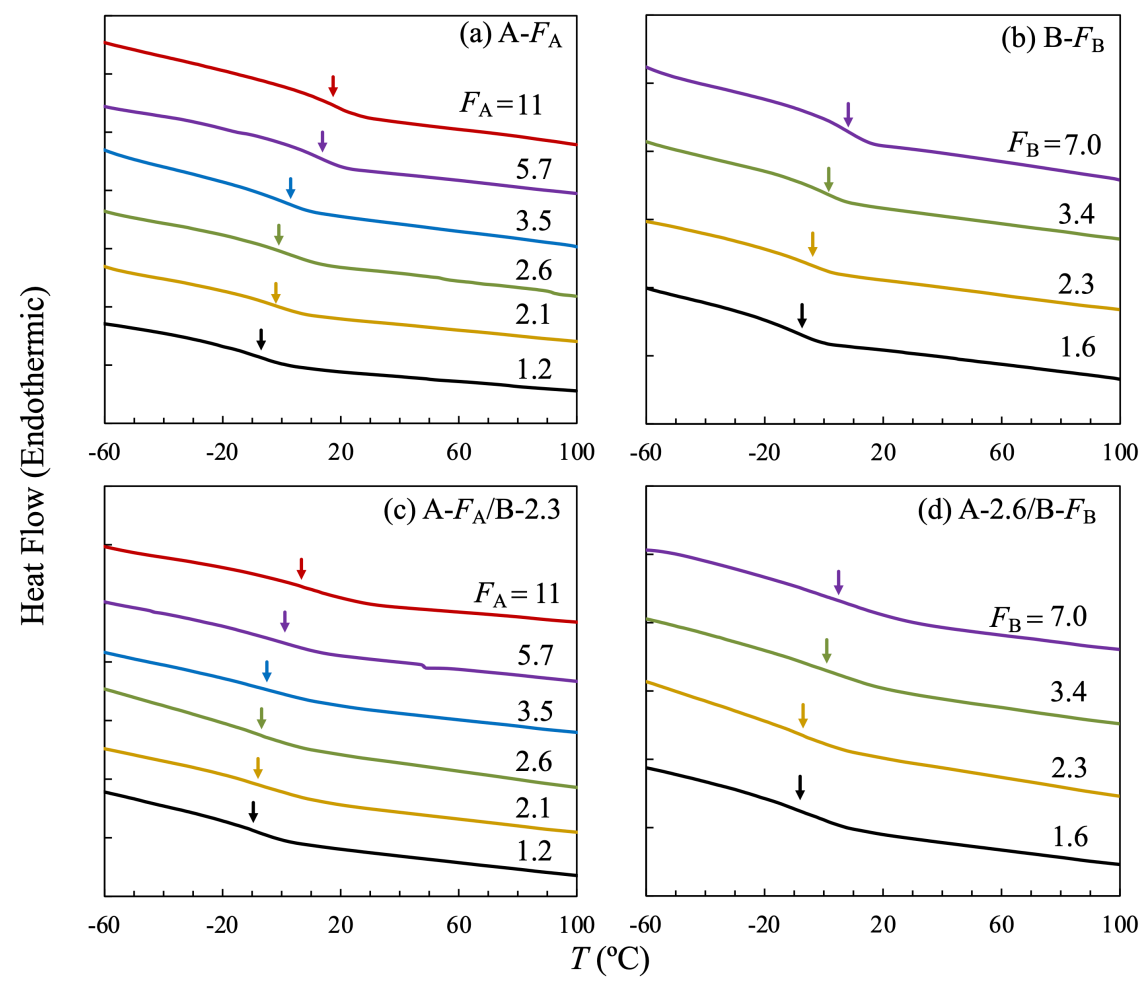

Figure S11. DSC traces of precursor samples (a) A- $F_{\mathrm{A}}$, (b) B- $F_{\mathrm{B}}$, and vitrimer samples (c) $\mathrm{A}-F_{\mathrm{A}} / \mathrm{B}-2.3$ and (d) A-2.6/B- $F_{\mathrm{B}}$.

\section{Linear Viscoelasticity}




\subsection{Stress relaxation measurements of vitrimer samples}

In order to reduce the measurement time at $180^{\circ} \mathrm{C}$ to better protect the sample, we performed stress relaxation measurements upon applying a step strain of $\gamma=5 \%$, which is within the linear regime, to obtain the relaxation modulus $G(t)$ as a function of time $t$. Figure S12 plots $G(t)$ against time $t$ for vitrimer samples (a) A- $F_{\mathrm{A}} / \mathrm{B}-2.3$ and (b) A-2.6/B- $F_{\mathrm{B}}$, the curves are fitted to a sum of Maxwellian modes:

$$
G_{0}(t)=\Sigma_{\mathrm{p}} g_{\mathrm{p}} \exp \left(-t / \tau_{\mathrm{p}}\right)
$$

We further transferred $G(t)$ into $G^{\prime}$ and $G^{\prime \prime}$ using $g_{\mathrm{p}}$ and $\tau_{\mathrm{p}}$ obtained from the fits:

$$
\begin{aligned}
& G^{\prime}=\sum_{\mathrm{p}} g_{\mathrm{p}} \omega^{2} \tau_{\mathrm{p}}^{2} /\left(1+\omega^{2} \tau_{\mathrm{p}}^{2}\right) \\
& G^{\prime \prime}=\sum_{\mathrm{p}} g_{\mathrm{p}} \omega \tau_{\mathrm{p}} /\left(1+\omega^{2} \tau_{\mathrm{p}}^{2}\right)
\end{aligned}
$$

The $G^{\prime}$ and $G^{\prime \prime}$ thus-obtained agree well with those obtained from the frequency sweep measurements.
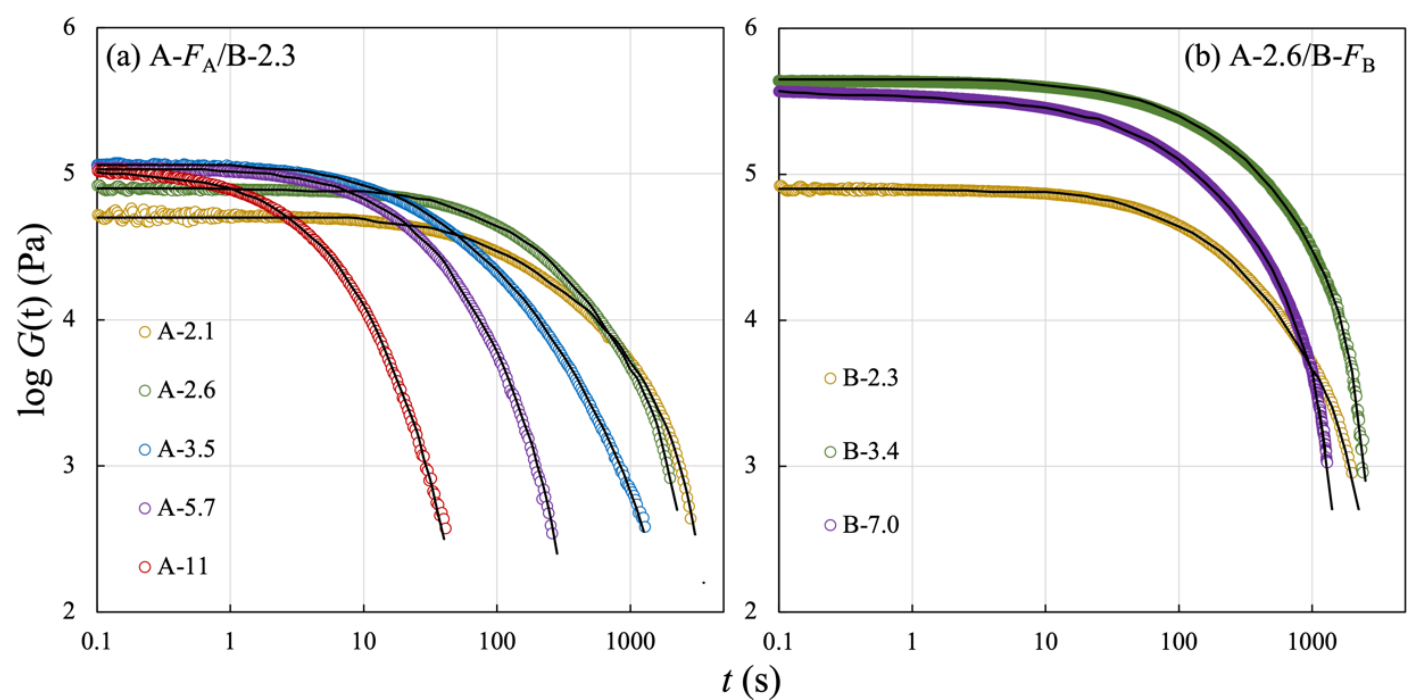

Figure S12. (a) Stress-relaxation measurements of A- $F_{\mathrm{A}} / \mathrm{B}-2.3$ samples at $180^{\circ} \mathrm{C}$. (b) Stress-relaxation measurements of A-2.6/B- $F_{\mathrm{B}}$ samples at $180^{\circ} \mathrm{C}$.

The stress relaxation modulus $G(t)$ of A-11/B-2.3 samples at $180^{\circ} \mathrm{C}$ was fit to a single Maxwell 
model, $G(t)=G_{0} \exp (-t / \tau)$ with modulus $G_{0}$ and relaxation time $\tau$ as two fitting parameters, as shown in Figure S13. $G(t)$ shows relaxation mode distribution broader than that expected from the single Maxwell model, which is consistent with the trend reported in the literature. ${ }^{3,4}$

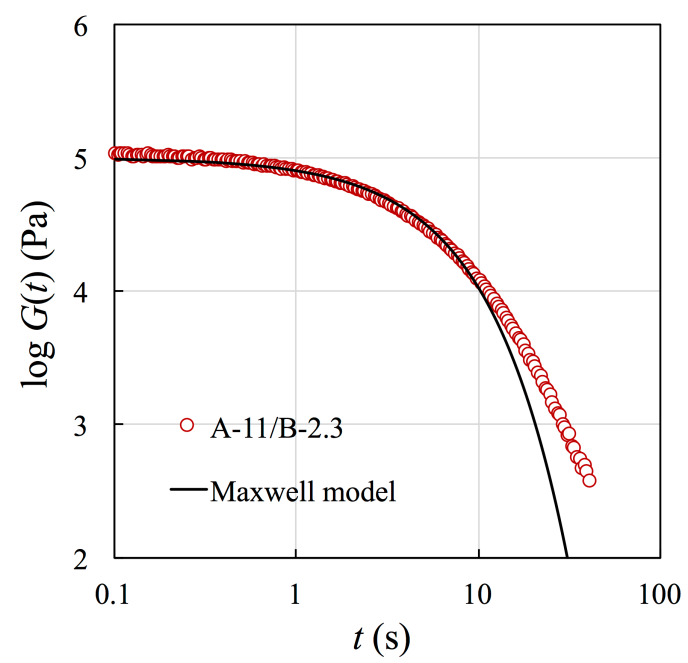

Figure S13. Stress relaxation modulus $G(t)$ plotted against $t$. for A-11/B-2.3 at $180^{\circ} \mathrm{C}$. The curve is fit to the Maxwell model.

\subsection{Frequency sweep measurements of A-2.6/B- $F_{\mathrm{B}}$ samples}

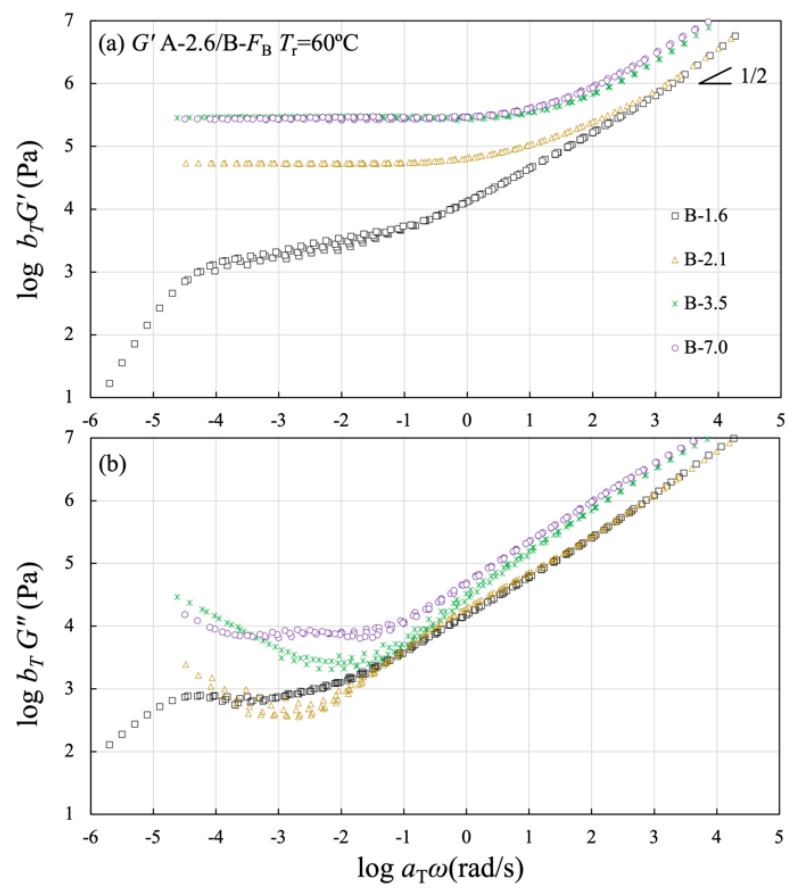

Figure S14. Test of time-temperature superposition of the A-2.6/B- $F_{\mathrm{B}}$ vitrimer samples with the values of $F_{\mathrm{B}}$ as indicated at $T_{\mathrm{r}}=60^{\circ} \mathrm{C}$. The storage and loss moduli (a) $G^{\prime}$ and (b) $G^{\prime \prime}$ from different temperatures are shifted under the guidance of Rouse part of modulus. 


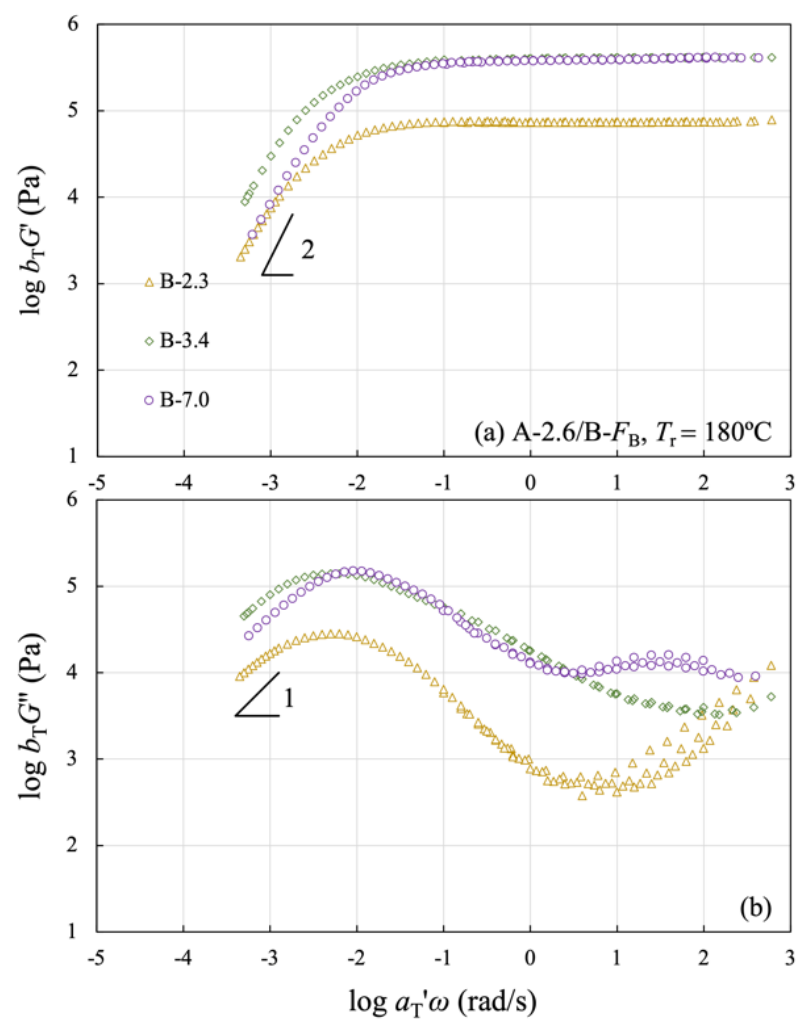

Figure S15. Test of time-temperature superposition of the A-2.6/B- $F_{\mathrm{B}}$ vitrimer samples with the values of $F_{\mathrm{B}}$ as indicated at $T_{\mathrm{r}}=180^{\circ} \mathrm{C}$. The storage and loss moduli (a) $G^{\prime}$ and (b) $G^{\prime \prime}$ from different temperatures are shifted under the guidance of network relaxation part of modulus.

\subsection{Shift factors of $\mathrm{A}-2.6 / \mathrm{B}-F_{\mathrm{B}}$ samples}
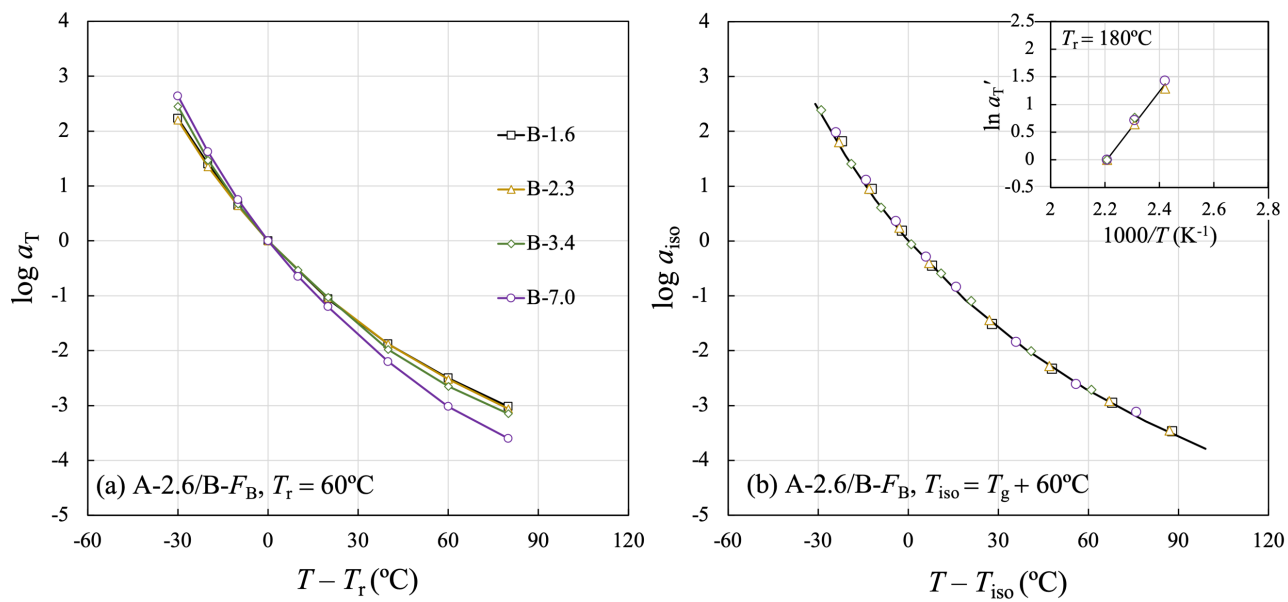

Figure S16. (a) LVE shift factor $a_{\mathrm{T}}$ in Figure S14, obtained from superposing the high- $\omega$ Rouse part of modulus, plotted against $T-T_{\mathrm{r}}$. (b) $a_{\text {iso }}$, reevaluated with respect to the new reference temperature $T_{\text {iso }}=T_{\mathrm{g}}+$ $60^{\circ} \mathrm{C}$, plotted against $T-T_{\text {iso. }}$. Inset shows the plots of $a_{\mathrm{T}}{ }^{\prime}$, which is obtained from superposing the low- $\omega$ network relaxation part of modulus in Figure S15, against 1000/T for the A-2.6/B- $F_{\mathrm{B}}$ vitrimer samples. 


\section{References}

1. Wang, Z.; Gu, Y.; Ma, M.; Chen, M. Strong, Reconfigurable, and Recyclable Thermosets Cross-Linked by Polymer-Polymer Dynamic Interaction Based on Commodity Thermoplastics. Macromolecules 2020, 53 (3), 956-964.

2. Wu, S.; Zhang, Z.; Chen, Q. Effects of alkyl group size on the structure and dynamics of poly(n-alkyl methacrylate)-based ionomers. Journal of Rheology 2019, 63 (6), 977-990.

3. Porath, L. E.; Evans, C. M. Importance of Broad Temperature Windows and Multiple Rheological Approaches for Probing Viscoelasticity and Entropic Elasticity in Vitrimers. Macromolecules 2021, 54 ( 10), 4782-4791.

4. Zhou, Y.; Groote, R.; Goossens, J. G. P.; Sijbesma, R. P.; Heuts, J. P. A. Tuning PBT vitrimer properties by controlling the dynamics of the adaptable network. Polymer Chemistry 2019, 10 (1), 136-144. 